\title{
Clinical treatment options for patients with homonymous visual field defects
}

\author{
Alison R Lane \\ Daniel T Smith \\ Thomas Schenk \\ Cognitive Neuroscience Research \\ Unit, Durham University, Durham, UK
}

\begin{abstract}
The objective of this review is to evaluate treatments for homonymous visual field defects (HVFDs). We distinguish between three treatments: visual restoration training (VRT), optical aids, and compensatory training. VRT is both the most ambitious and controversial approach, aiming to restore portions of the lost visual field. While early studies suggested that VRT can reduce the visual field defect, recent studies using more reliable means of monitoring the patients' fixation could not confirm this effect. Studies utilizing modern optical aids have reported some promising results, but the extent to which these aids can reliably reduce the patients' visual disability has yet to be confirmed. Compensatory approaches, which teach patients more effective ways of using their eyes, are currently the only form of treatment for which behavioral improvements have been demonstrated. However, with the exception of one study using a reading training, placebo-controlled clinical evaluation studies are lacking. It is also not yet clear whether the training benefits found in laboratory tasks lead to reliable improvements in activities of daily living and which of the various forms of compensatory training is the most promising. It is therefore too early to recommend any of the currently available treatment approaches.
\end{abstract}

Keywords: homonymous hemianopia, rehabilitation, treatment outcome

\section{Introduction}

The visual field is the entire space in which visual stimuli can be perceived when the eyes are fixating. If someone has a visual field defect this means that they have lost the ability to see visual stimuli which are presented in one part of the visual field. With homonymous visual field defects (HVFDs), the same part of the visual field is affected for both the right and left eye. Approximately $20 \%$ of people with acquired brain injury may develop a HVFD (Kasten et al 1999), which occurs following damage to the neural visual pathway, specifically damage posterior to the optic chiasm. Homonymous hemianopia (see Figure 1), in which one half of the visual field is blind, occurs in approximately $75 \%$ of cases (Zihl 1995a).

HVFDs can be very disabling. They impair the patient's ability to obtain a complete visual overview (Zihl 1995a), which can impact on their ability to interact with their environment. In addition, patients can experience further impairment in their daily life; for example, driving is prohibited in the majority of cases (Kooijman et al 2004) and HVFDs can lead to severe reading difficulties (Leff et al 2001). Such difficulties arising from visual problems can have additional effects on the social and emotional functioning of the individual.

Traditionally HVFDs were considered untreatable, but recent advances in our understanding of the neural capacities for functional reorganization has led to an upsurge of attempts to achieve a reduction of the field loss through training. At the same time new rehabilitation procedures have been studied which might allow patients to more efficiently perceive the whole visual world and to improve reading 


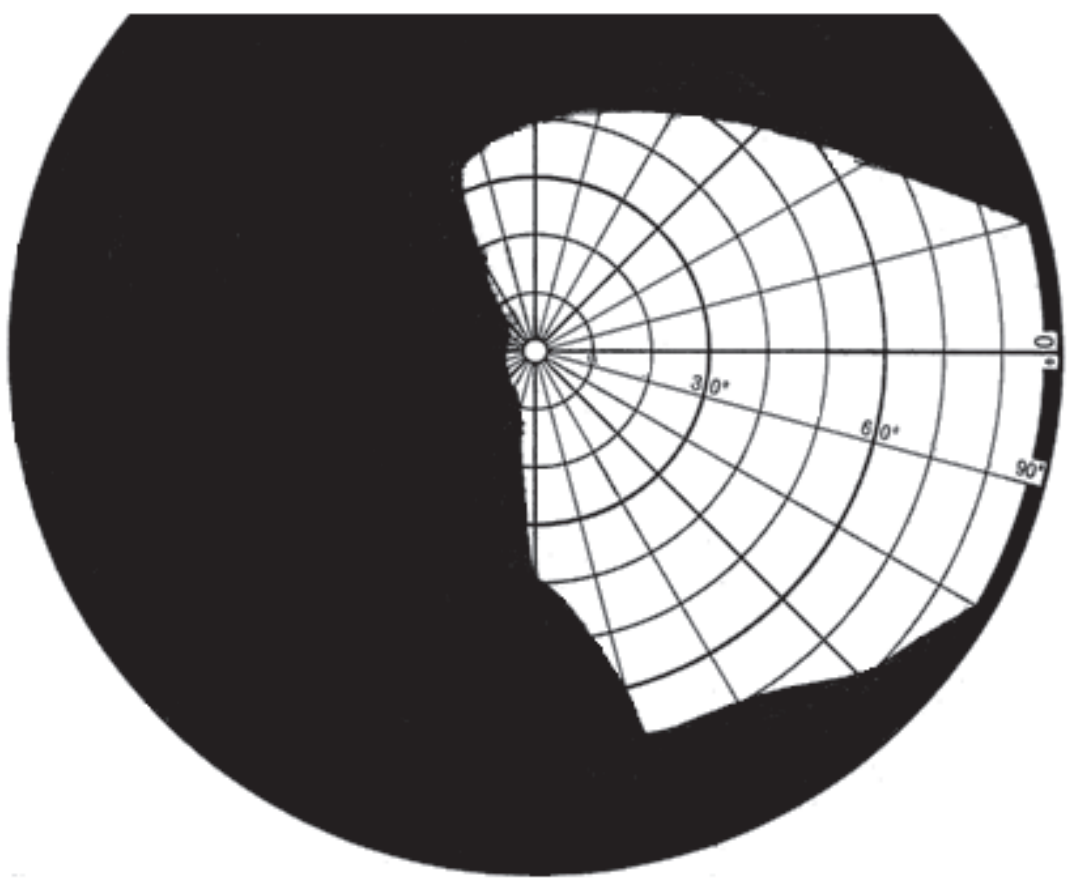

Figure I Binocular visual field plot representing a left-sided hemianopia.

performance despite persistent HVFDs. These procedures have been reviewed previously (Kerkhoff 2000; Pambakian et al 2005; Pelak et al 2007), including a recent systematic review (Bouwmeester et al 2007), and it was concluded that compensatory rehabilitation procedures can improve the visual searching skills and reading performance of patients with HVFDs. Despite these encouraging results, patients often do not receive specific rehabilitation for their HVFDs (Kerkhoff 1999; Pambakian et al 2005). Therefore the issue of how to increase rehabilitation needs to be addressed.

This review will examine what is needed to make the behavioral treatment of HVFDs more clinically relevant. Firstly, we will address why patients with HVFDs need treatment. Secondly, we will evaluate the efficacy of the different treatments which have been developed. Finally, we will discuss unresolved issues which may currently prevent clinicians from implementing the rehabilitation strategies.

\section{HVFD: Impairment and disability}

Reading problems have been objectively observed in $48 \%$ of hemianopic patients (Zihl 1999a) and are often cited as the most relevant behavioral difficulty (Kasten et al 1999). Hemianopic dyslexia is the term used to describe the particular pattern of reading problems that are associated with HVFDs, and the specific reading difficulties depend mainly on HVFD location (Trauzettel-Klosinski and Brendler 1998). For example, in Western cultures where text is read from left to right, patients with right-sided defects experience more severe problems since the parafoveal information to the right is crucial for guiding appropriate reading eye-movements. Furthermore, reading is typically worse in patients who have less than $5^{\circ}$ of visual sparing (Zihl 1995b), therefore specifically patients with macular splitting or a central scotoma. However, such patients are the minority of cases (Leff 2004). Analysis of patients' eye-movement data indicates that patients with HVFDs do not appear to compensate for their field loss when reading and show inefficient eye-movements (Trauzettel-Klosinski and Brendler 1998; McDonald et al 2006), although this is not the case for all patients (Gassel and Williams 1963). Gassel and Williams (1963) also found that some patients with atypical eye-movements can still exhibit adequate reading speeds, demonstrating that atypical eye-movement patterns during reading do not necessarily lead to an impaired reading ability.

HVFDs can also lead to difficulties creating a complete visual overview, particularly in novel environments, thereby affecting the ability to find objects. This ability has been examined using visual search tasks, where the aim is to locate a target item amongst distracting elements. Patients typically take longer than healthy individuals to complete such tasks (Zihl 1995a), and can have similar difficulties performing other visual tasks such as identification and sorting (Zihl and Wohlfarth-Englert 1986; Zihl et al 1988). Many patients with HVFDs execute abnormally short eye-movements 
when looking into their blind regions and each movement is typically slower than that of a normally-sighted individual. This strategy is known as saccadic hypometria (Zihl 2000) and limits the patients' ability to effectively search their environment, which contributes to their disorientation and obstacle avoidance problems. Approximately $70 \%$ of patients show such disorganized searching strategies (Kerkhoff 1999).

Due to the enjoyment which can be gained from reading and other leisure activities requiring visual search skills, any impairment in these has obvious consequences for the emotional well-being of the patient (Stelmack 2001). Similarly, HVFDs can restrict many other activities such as driving, which can lead to a loss of independence, thereby affecting social and emotional functioning. The primary cause of HVFD is stroke (Huber 1992) and accordingly most patients are elderly (Cairns 2004). Therefore, visual problems increase the risk of accidents such as falls, to which this age-group are already prone (Anderson 2002). HVFDs also reduce the efficacy of other rehabilitation procedures which may be aimed at increasing the patient's mobility (for example physiotherapy). Bearing these factors in mind many patients have low scores in activities of daily living (ADL) measures (Patel et al 2000; Sànchez-Blanco et al 2002), and functional rehabilitation outcomes are poor (Reding and Potes 1988).

\section{Spontaneous recovery}

Some spontaneous visual field restoration is widely accepted to occur. However, the number of patients who experience any restoration is undetermined, with reports ranging from $7 \%$ to $85 \%$ of cases (Kasten et al 1999). The amount of field recovery that an individual can experience is similarly variable (Zihl and Kennard 1996). In an extensive review of spontaneous recovery, Zhang and colleagues (2006) observed that the degree of natural recovery decreased as the amount of time since the onset of the HVFD increased. The approximate maximal period of spontaneous recovery is typically 3 months (Pambakian and Kennard 1997). In summary, spontaneous visual field recovery does not occur in all patients and complete recovery is rare, therefore rehabilitation for such patients is important. Knowing the likely pattern of natural recovery is important for assessment and rehabilitation, and is useful for determining the time at which training will be maximally effective.

Patients may also try to adapt to their visual loss. The obvious way of compensating for HVFDs is to make larger and more frequent eye-movements, specifically into the blind areas. Unfortunately not all patients adopt this strategy
(Kerkhoff 1999). Rather, several researchers have shown that the eye-movements of many HVFD patients are very small and their scan-paths (the pattern of eye-movements used to scan a complex visual representation) are disorganized (Meienberg et al 1981; Zihl 1995a, 1999b; Pambakian et al 2000). Patients who have a chronic HVFD show more organized scanning strategies than those patients whose difficulties are a recent occurrence (Zihl 1995a; Pambakian et al 2000). However, many patients' eye-movements are still disorganized 14 months after onset (Kerkhoff 1999). These abnormal eye-movements can also be observed during reading (Zihl 1995b).

Patients may also use other forms of behavioral compensation. One method is the use of eccentric fixation; the eye is rotated slightly towards the blind hemi-field rather than straight ahead (Gassel and Williams 1963). This means that the centre of the observed image does not fall exactly on the fovea, but instead the image falls further into the seeing field at a slightly eccentric position. This strategy is found in approximately $30 \%$ of cases and can increase reading speed (Trauzettel-Klosinski 1997), although the impact of such a strategy on other activities has not been clearly determined. It is a strategy which may be of use for patients with little central vision, for example those with macular splitting or a central scotoma, which as already mentioned is the minority of patients (Leff 2004). However, eccentric fixation is not a useful strategy for patients with an intact fovea. Another strategy witnessed in children involves to-and-fro rocking motions, to bring greater portions of the visual field into view (Boyle et al 2005).

In summary, HVFDs are debilitating and the sensory loss is exacerbated by the adoption of slow and inefficient search strategies when exploring the blind field. The prognosis for spontaneous recovery appears poor, and although adaptation is possible many patients do not develop effective ways of compensating for their deficits. Specific interventions are required for these patients.

\section{Intervention}

Most research has focused on three treatment approaches: restorative training, optical aids, and compensatory training. The restorative approach is the most ambitious, aiming to reduce the visual field loss through prolonged training. The second approach uses optical aids to artificially expand the patient's visual field such that parts of the visual world which would otherwise fall into the blind field now appear in the seeing field. The third approach is compensatory training. This therapy is based on the assumption that the 
visual field defect cannot be changed significantly, and therefore attempts to alleviate the resulting disability by teaching patients to make more efficient eye-movements. We will discuss each of these three rehabilitation strategies in separate sections.

\section{Restorative training}

Restorative training aims to restore vision (at least in part) to the blind visual field, based on evidence which supports plasticity in the visual system of both animals (Cowey and Weiskrantz 1963; Cowey 1967; Mohler and Wurtz 1977; Eysel and Schwiegart 1999) and humans (Donoghue 1997). Whilst there was early promise for the potential of such training (Zihl and Von Cramon 1979; Zihl and Von Cramon 1985), some dismissed the approach as ineffective, with any supposed field increase being regarded as the product of eyemovements (Balliet et al 1985).

Restorative training was later revived by Kasten, Sabel, and colleagues who introduced a computerized therapy called Vision Restoration Therapy (VRT; Kasten and Sabel 1995; Kasten et al 1997). During VRT patients fixate a central point whilst visual stimuli are repeatedly presented in the border region between the blind and seeing field (the transition zone). The training is typically conducted in daily one-hour sessions for 6 months. Placebo-controlled studies have suggested that VRT leads to significant increases in the visual field (Kasten et al 1998, 2000), although more so for patients with optic nerve as opposed to cortical damage. These field increases were still observed at least 6 months after the end of the treatment (Kasten and Sabel 1995; Kasten et al 2001).

However, despite the apparent success of VRT critics have challenged the claim that it is an effective treatment (Horton 2005; Plant 2005; McFadzean 2006). This is because the claims of significant field increases found in the VRT studies mentioned above were based on a method of assessing the visual field which was incorporated into the training device and did not allow a reliable way of controlling the patients' fixation. The effect of VRT has been re-evaluated using techniques which allowed a much more reliable means of monitoring the patients' fixation, specifically the use of a scanning laser ophthalmoscope (SLO; Jamara et al 2003) combined with microperimetry. In studies whereby they reliably monitored fixation during visual field assessment no significant visual field increases were obtained (Sabel et al 2004; Reinhard et al 2005; Schreiber et al 2006).

Recently Kasten and colleagues (2006) responded to this challenge by publishing a study in which they used a video-based system to monitor their patients' fixation and found a significant (but somewhat modest) field increase of 1.8 degrees. This finding is insufficient to re-establish the therapeutic value of VRT for two reasons. Firstly, because the employed fixation-control technique is inferior to that used by other studies which did not find significant field increases. Secondly, even if we accept the reported field increase, such an increase of $1.8^{\circ}$ is clearly not enough to convey a clinically significant benefit to patients who have endured 3 months of daily training and invested a considerable amount of money. In this context it is important to note that these expenses are currently not covered by medical insurance companies (Pelak et al 2007).

Even if the claim that VRT produces a clinically-relevant field increase is dismissed, the widespread subjective improvements reported by many participants of this training cannot be disregarded (Mueller et al 2003; Sabel et al 2004). The fact that improvements are sometimes reported by patients in the absence of any training-induced field recovery (Mueller et al 2003) raises an interesting question: why do patients report that subsequent to the training they find it easier to find objects and avoid obstacles, if their visual field is unchanged? It might be that these reports simply reflect the patients' desire to justify a training in which they have invested a lot of time, effort, and money. However, it is also possible that VRT leads to behavioral improvements. For example, it is possible that VRT cues patients to allocate more attention into their blind field, and such attentional cueing can improve target detection (Poggel et al 2006). Although visual costimulation was found to be no more effective than single stimulation VRT at expanding the visual field, it is clear that VRT has beneficial effects on attentional performance (Kasten et al 2007). Further research into the role of attention for visual field rehabilitation is required.

In addition to possible effects on attention, VRT could also inadvertently lead to eye-movements being more frequently directed into the blind field, although this explanation is denied by Kasten and colleagues (2006). However, it could explain why restorative training leads to changes in cortical activity as reported by several small-sample ( $\mathrm{n}=$ 1-5) imaging and electrophysiology studies (Julkunen et al 2003, 2006; Pleger et al 2003), since saccadic changes due to compensatory mechanisms could influence widespread neural activity. Initially these changes in cortical activity were interpreted as evidence for the training-induced brain plasticity underlying the recovery of visual field loss. However, in the absence of reliable evidence for such visual field recovery this interpretation appears unlikely. 
More encouraging results have been obtained with children. Werth and colleagues presented findings from randomized placebo-controlled trials with a restorative training used with children aged between 1 and 15 years (Werth and Moehrenschlager 1999; Werth and Seelos 2005). In some cases the visual field defect disappeared completely, and the mean increase was 65 degrees. Such dramatic increases coincide with evidence suggesting that there is greater potential for recovery from damage sustained early in life (Payne et al 1996; Boyle et al 2005), possibly because child and adult cases typically differ with regards to etiology and lesion location (Kedar et al 2006), or perhaps due to greater neuronal plasticity more generally in children. However, due to the age of these patients conventional perimetry could not be performed and instead the researchers had to rely on observed changes in target-directed eye-movements to estimate the extent of visual field recovery. Accordingly the visual field measurements and reported field increases are difficult to interpret and could reflect compensatory mechanisms rather than restorative ones. Given the large improvements which appear possible, this training would appear worthwhile pursuing with children who have HVFDs.

In conclusion, restorative training in adults has failed to fulfill its early promise. Recent studies suggest that VRT does not lead to significant increases in visual field size but consistently yields subjective improvements. The basis of these subjective improvements is still unclear but it is possible that VRT leads to compensatory changes in behavior which are as yet unconfirmed. However, even if we were to assume that VRT leads to significant behavioral improvements it would still be inferior to other forms of compensatory training (see below) which appear to produce effective behavioral compensation with significantly less effort, cost and time.

\section{Optical aids}

Optical aids such as prism glasses can be used to reduce the apparent visual field loss by shifting visual stimuli from the blind field into the patient's seeing field. These prisms are fitted to spectacles but need to be restricted to just one half of each of the lenses (typically on the side of the blind field). If the prisms were fitted across the entire lens then the visual space corresponding to the unaffected side would be moved outside the field of view, thereby simply replacing one blind field with another. Such prisms can be fitted to just one eye (monocular sector prisms) or both eyes (binocular sector prisms). Whilst such aids appear to enhance visual functioning (Gottlieb et al 1998; Lee and Perez 1999; Szlyk et al 2005) they have their limitations. Monocular prisms provide an expansion of the visual field but at the cost of creating central double-vision (diplopia) which patients experience as unpleasant. Binocular prisms lead to field relocation rather than field expansion. Such problems probably explain why so far these optical aids proved only moderately successful in HVFD rehabilitation.

Peli $(2000 ; 2001)$ has introduced a new set of spectacles with monocularly fitted sector prisms which extend across the entire width of the spectacle lens but which spare the central aspect. This is known as vision multiplexing and using this technique field expansion is achieved without central diplopia. Peli (2000) reported field expansion of about $20^{\circ}$ and noted on the basis of subjective reports that patients seemed to benefit from the spectacles. Vision multiplexing seems promising but randomized controlled trials using objective measures of functional improvement are required to evaluate the clinical potential of such a technique.

\section{Compensatory training}

Even if training cannot achieve a significant reduction in the visual field loss, it might still be possible to help patients to cope more effectively with their HVFD. Scanning the visual world systematically with large sweeping eye-movements would appear to be the most obvious form of compensation, however many patients do not spontaneously adopt this strategy (Kerkhoff 1999). In order to improve patients' ability to compensate for their visual loss, several researchers have developed training schemes designed to teach patients more efficient strategies for visual scanning.

The compensatory training approaches typically use target-localization tasks to train patients to make large eyemovements and use visual search tasks to teach patients to use systematic scanning strategies when searching their visual world. Sometimes additional training is included which helps the patient to utilize the strategies in everyday situations such as crossing the street or finding objects around the home (Kerkhoff et al 1992, 1994; Pambakian et al 2004). With compensation training patients usually receive daily one hour training sessions for about four weeks making it much less demanding than VRT.

Compensatory training in general leads to improved search performance and efficiency (Kerkhoff et al 1992, 1994; Zihl 1995a; Nelles et al 2001; Pambakian et al 2004; Verlohr and Dannheim 2007). Two studies confirmed that the improvements obtained after training were significantly greater than those observed during untrained periods and are thus training specific (Kerkoff et al 1994; Pambakian et al 2004). For example, Pambakian and colleagues (2004) 
found that $76 \%$ of patients had faster search times following training, while 14\% remained unchanged, and 10\% were actually slower. Those with slower search times showed an improvement in their detection rates. Compensatory training has also been found to significantly enlarge the search field (Kerkhoff et al 1992, 1994; Pambakian et al 2004). Additionally, studies using eye-tracking show that compensatory training leads to better organized scanning strategies and larger saccades (Zihl 1995a). Furthermore, it was found that the post-training search improvements could be maintained for at least one month, and in some cases up to 22 months (Kerkhoff et al 1992).

Not only does it appear from these studies that compensatory training can significantly improve search but it is possible that it may actually increase the visual field itself (Kerkhoff et al 1992, 1994). However, not all studies have found significant visual field increases after compensatory training (Zihl 1995; Nelles et al 2001; Pambakian et al 2004). Caution is required when interpreting these findings as the same limitations relating to fixation control during perimetry are present as in studies examining restorative approaches.

The reading performance of patients with HVFDs is also often impaired (Leff et al 2001). Consequently, specific compensatory reading training procedures have been developed to directly address this deficit (Zihl 1995b; Han et al 2004; Spitzyna et al 2007). Zihl (1995b) showed that such training can improve reading accuracy and speed although unfortunately there was no control group and so it is not known to what extent the effects were due to the training provided. In contrast, a recent study by Spitzyna and colleauges (2007) included a placebo training to assess the specific effects of a reading training for patients with hemianopic dyslexia. They tested 19 patients with right-sided hemianopia and divided them into two groups. Group 1 received a reading training for two 4-week blocks. During this training, patients practiced reading moving text which scrolled from right to left. In group 2 patients received a 4-week block of placebo training and a 4-week block of reading training. In the placebo-training patients received pairs of pictures which differed only in a number of minor features and they had to detect these differences. The reading training but not the placebo training induced significant improvements in reading speed.

The results surrounding compensatory training indicate the promise which such an approach holds for helping patients to adapt to their visual loss, and whilst controversy continues to surround the use of VRT, compensation would appear to be a viable rehabilitation option for the patients with HVFDs. However, it is important to note that for most compensatory training regimes a placebo-controlled study examining their efficacy is still missing.

\section{Unresolved issues of HVFD rehabilitation}

As described above the lack of placebo-controlled evaluation trials (for an exception see Spitzyna et al 2007) means that the clinical efficacy has not yet been established for any of the described rehabilitation procedures. It is thus too early to give a firm recommendation for any of these approaches. However, the compensatory training approach is the only one for which behavioral improvements in the form of improved search times, increased reading speed and larger eye-movements have been demonstrated. In fact, for one form of compensatory reading training its superiority over a placebo-training has already been established (Spitzyna et al 2007). In contrast the same is not true for either restorative training or optical aids. In the case of restorative training the early claims of increased visual field size following training have not been confirmed by studies using more reliable means of assessing the visual field size and there is currently no evidence that the reported subjective improvements correspond to measurable behavioral improvements. In the case of optical aids evidence for behavioral improvements are also lacking, in particular for their most promising forms, the vision multiplexing prisms. Thus on the basis of current evidence the compensatory approach appears to be in our view the most promising, and we will therefore now turn our attention to those aspects of the compensatory therapy which require further research.

\section{Transfer of training benefits to activities of daily living}

The first issue relates to the question of whether the achieved training gains also lead to relevant improvements in activities of daily living (ADL), an aspect which is crucial to the clinical evaluation of any rehabilitation procedure. Unfortunately most studies examining compensatory search training either do not assess its impact on ADL tasks or rely solely upon subjective reports. Using questionnaires several researchers have found that patients do report improvements in such activities as finding objects in a room or on a table, and crossing the street (Kerkhoff et al 1994; Nelles et al 2001; Pambakian et al 2004), indicating that compensatory training produces functional improvements. It is however important to establish the functional benefits of the training with behavioral measures since subjective gains can be unreliable indicators of rehabilitation success. 
There are a few studies which have used objective measures to assess the transfer of training benefits to ADL tasks. Kerkhoff and colleagues (1994) demonstrated that combined compensatory search training (ie, training which combines search tasks and exercises such as finding objects around the home) can yield improved search performance in more naturalistic forms of visual search (eg, searching for an item amongst distracters on a table) which use a wider field of view than that used during the training. Following training the patients in this study showed a 50\% reduction in search time on the table test. Further to this, they also reported that $91 \%$ of the sample returned to some sort of part-time work after the training, indicating a positive functional outcome. Pambakian and colleagues (2004) reported training-related improvements in performance on activities representing ADL tasks, such as threading beads onto string. Whilst this does indicate the successful transfer to the visuomotor domain of the training benefits, there is a question of how much such tasks actually tell us about improvements in more commonplace everyday activities. Future work should examine more closely the efficacy of the training in relation to more relevant examples of ADL.

Driving is a major activity for which transfer of training gains would be beneficial since it is prohibited for the majority of patients with HVFDs. Unfortunately Kooijman and colleagues (2004) found that only 2 out of the 17 homonymous hemianopic patients who failed a test of practical driving fitness passed this test after a form of compensatory training. Given the social and emotional impact that the loss of driving has on patients with HVFDs the effect that compensatory training has on driving ability should be further examined. It is worth noting that patients with HVFDs may be able to drive as adequately as normal, healthy individuals (Schulte et al 1999) and therefore perhaps driving guidelines should be modified such that an HVFD is not an automatic cause for license revocation.

Nelles and colleagues (2001) reported that patients' subjective impression of their reading ability had improved after the compensatory search training. However, since reading performance has not been measured it is not possible to conclude if there is transfer from search training to reading tasks. Again reading is an activity which many patients report having difficulties with and which can severely impact on their quality of life, and as such should be considered an important outcome measure in future research. If it is found that general compensatory training can benefit reading (or that reading training can benefit other everyday activities) then only one type of training would be required.
Currently there is insufficient information about how the gains achieved with compensatory training transfer to other relevant activities like driving, reading, visuomotor control, and visual searching in natural surroundings. Furthermore, it is currently unclear whether some ADL tasks benefit more from training than others. If it is confirmed that some ADL tasks do not benefit sufficiently from compensatory training (for example reading), then training which addresses the specific requirements of those tasks will be needed.

\section{Predictors of good training outcome}

Not all patients benefit from compensatory training. In order to maximize its efficacy it is necessary to identify factors that contribute to the success or failure of this training. Conventional predictors of rehabilitation outcome, which include the cause of the HVFD (etiology) and the time since onset of the HVFD when training takes place have been examined. Etiology does not seem to have a significant effect on outcome (Kerkhoff et al 1992, 1994), although this is hardly surprising given that most patients share the same etiology, namely stroke. With respect to the timing of training, the results are mixed as some studies suggest that earlier training is more beneficial (Zihl 1995a), whilst others fail to find such an association (Kerkhoff et al 1992, 1994). Several studies have failed to find any association between age and training outcome (Kerkhoff et al 1992, 1994; Zihl 1995a). Kerkhoff and colleagues (1992) found that those patients who initially had the severest visual problems were those who showed the largest improvements after the training, thus pre-training impairment level could predict the possible success of the training.

A very plausible predictor for good training outcome is the degree of spared visual ability in the blind field. Some patients can respond quite accurately to visual stimuli presented to their blind field (for example by pointing to it) even though they insist that they cannot see it. This phenomenon has been called blindsight (Weiskrantz et al 1974) and it is estimated that $15 \%-20 \%$ of HVFD patients show this (Blythe et al 1987). It has been suggested that training patients to be aware of their blindsight capacity could be a useful rehabilitation strategy (Boyle et al 2005) and repeatedly testing blindsight can lead to improved blindsight performance (Zihl 1980). It seems plausible that patients with blindsight are more successful in making accurate saccadic eye-movements to targets in their blind field and could therefore benefit more from compensatory training. However, this prediction has not yet been tested.

Cognitive variables, such as the patient's ability to allocate visual attention or their spatial memory capacity, which 
might contribute to the success or failure of compensatory training, have not yet been examined. In summary, it seems clear that although compensatory treatments benefit the majority of participants, the factors which predict successful treatment outcome on an individual basis remain unclear.

\section{Comorbidities}

Some previous studies examining compensatory training have excluded people with HVFDs who suffered from additional disorders such as oculomotor deficits or hemispatial neglect. Disorder comorbidities are common (Anderson and Rizzo 1995) and patients with multiple difficulties are typically more functionally impaired than those with a single defect (Patel et al 2000). The impact that this may have on rehabilitation has not been fully established.

Hemispatial neglect is a disorder which typically occurs after right-hemispheric damage and leads to patients ignoring sensory information from the contralesional half of their body or surroundings. It might be predicted that patients with such additional disorders will gain less from compensatory training. However, some authors have argued that patients with both HVFD and hemispatial neglect can benefit from the training but require a more intensive schedule (Kerkhoff et al 1992, 1994). Similarly it has been shown in a case-study that a patient with multiple visual problems such as amblyopia and impaired form vision could still benefit from compensatory training (Hiramaya et al 2004).

In summary, HVFD patients with comorbidities such as hemispatial neglect may require more intensive training, but may still benefit from compensatory training. Having said that, the current evidence is scant and rigorous studies comparing the training benefits in large samples of patients with and without such co-morbidities have yet to be conducted.

\section{Parameters of effective compensatory training}

Although it has been shown that compensatory training in general can lead to significant functional gains (Pambakian et al 2004), a number of different training regimens have been used and it is unclear which is the most effective. These regimens differ with respect to the required effort and cost, and it is important to establish whether the simpler and less costly forms are as effective as the more laborious ones. For example, training displays of various size have been used ranging from computer or television monitors, which train only the central 25 degrees of the visual field (Pambakian et al 2004), to displays which fill the entire visual field (Nelles et al 2001). It is obvious that training on a small screen is less costly because it means that the training can potentially be performed by the patient in their own home, as was done in the study by Pambakian and colleagues (2004), and as is the case with VRT (Kasten and Sabel 1995).

Treatment duration also differs significantly, ranging between 12 and 60 sessions (Kerkhoff et al 1992). In several studies (Kerkhoff et al 1992, 1994; Zihl 1995a) patients receive training until their performance plateaus or their search field increases by a specified amount. However in the other studies patients all receive a standardized amount of training. Research should attempt to determine what the maximal amount of training required is, and possible factors that may influence the amount of training required by a specific individual. This will ensure that time and resources are utilized to their best advantage.

Different types of training programs have not been compared directly and so we do not know yet whether the different regimens are equally effective. A confounding problem is that different outcome measures are also used in many of the studies making it difficult to compare them. Standardizing the outcome measures may aid this process, and Verlohr and Dannheim (2007) recently proposed the visual performance test as a standardized outcome measure for the purpose of assessing search times. This task involves patients having to visually locate as quickly as possible a series of targets which can be at one of eleven positions on a screen. Reaction time is the main outcome measure.

Another version of compensatory search training combined auditory cues with visual search displays and reported significant improvements in exploratory eye-movements and transfer to ADL (Bolognini et al 2005). However, it is not known whether the achieved gains are superior to those observed with training using purely visual displays. A direct comparison between conventional and combined (ie, visual plus auditory stimulation) training is required to determine if adding auditory cues increases the efficacy of compensatory training.

In summary, there are different versions of compensatory training available, varying specifically in relation to the size of the training stimuli, the duration of the training, and the addition of attentional aids. Currently it is unclear which version produces the best clinical outcome. Standardizing outcome measures will make it easier to directly compare the benefits of different training techniques, which will allow researchers to develop the maximally effective training paradigm.

\section{Conclusion}

With the exception of one form of a compensatory reading training (Spitzyna et al 2007) clinical efficacy has not been 
unequivocally established for any of the above described rehabilitation procedures. To establish clinical efficacy randomized placebo-controlled clinical evaluation trials are needed. Currently it is therefore too early to recommend any of the described rehabilitation procedures. However, we would like to argue that compensatory approaches have come further towards the aim of establishing their clinical efficacy than either the VRT approaches or the use of optical aids. In the case of compensatory approaches several studies found significant behavioral improvements following the training. The same can not be said for either VRT or optical aids. In both cases their claim of clinical efficacy currently rests on subjective patient reports and it is yet unknown whether these subjective reports of improvement correspond to measurable behavioral improvements. Apart from the need for placebo-controlled clinical evaluation trials we have also identified a number of other issues which need to be addressed by future research. These include the question of transfer, which is whether or not the compensatory training leads to improvements in relevant ADL tasks, the issue of outcome predictors and also which specific version of the compensatory training is the most effective form of treatment.

\section{Disclosure}

The researchers are supported by a grant from the Wolfson Research Institute and A. Lane is supported by a studentship jointly provided by the ESRC and MRC.

\section{References}

Anderson SW. 2002. Visuoperceptual Impairments. In: Eslinger PJ ed. Neuropsychological Interventions: Clinical Research and Practice. New York: The Guildford Press. pp. 163-81.

Anderson SW, Rizzo M. 1995. Recovery and rehabilitation of visual cortical dysfunction. NeuroRehabilitation, 5:129-40.

Balliet R, Blood KMT, Bach-y-Rita P. 1985. Visual field rehabilitation in the cortically blind? J Neurol Neurosurg Psychiatry, 48:1113-24.

Blythe IM, Kennard C, Ruddock KH. 1987. Residual vision in patients with retrogeniculate lesions of the visual pathways. Brain, 110:887-905.

Bolognini N, Rasi F, Coccia M, et al. 2005. Visual search improvement in hemianopic patients after audio-visual stimulation. Brain, 128:2830-42.

Bouwmeester L, Heutink J, Lucas C. 2007. The effect of visual training for patients with visual field defects due to brain damage: a systematic review. J Neurol Neurosurg Psychiatry, 78:555-64.

Boyle NJ, Jones DH, Hamilton R, et al. 2005. Blindsight in children: does it exist and can it be used to help the child? Observations on a case series. Dev Med Child Neurol, 47:699-702.

Cairns NJ. 2004. Neuroanatomy and Neuropathology. In: Goldstein LH, McNeil JE eds. Clinical Neuropsychology: A Practical Guide to Assessment and Management for Clinicians. Chichester: John Wiley and Sons Ltd. pp. 23-56.

Cowey A. 1967. Perimetric study of field defects in monkeys after cortical and retinal ablations. QJ Exp Psychol, 19:232-45.

Cowey A, Weiskrantz L. 1963. A perimetric study of visual field defects in monkeys. Q J Exp Psychol, 15:90-115.

Donoghue JP. 1997. Limits of reorganization in cortical circuits. Cereb Cortex, 7:97-9.
Eysel UT, Schweigart G. 1999. Increased receptive field size in the surround of chronic lesions in the adult cat visual cortex. Cereb Cortex, 9:101-9.

Gassel MM, Williams D. 1963. Visual function in patients with homonymous hemianopia. Part II: Oculomotor mechanisms. Brain, 86:1-36.

Gottlieb DD, Fuhr A, Hatch WV, et al. 1998. Neuro-optometric facilitation of vision recovery after acquired brain injury. NeuroRehabilitation, 11:175-99.

Han Y, Ciuffreda KJ, Kapoor N. 2004. Reading-related oculomotor testing and training protocols for acquired brain injury in humans. Brain Res Protocols, 14:1-12.

Hiramaya K, Sakai S, Yamawaki R, et al. 2004. Visual search training for a case of homonymous field defect with multiple visual dysfunctions [abstract]. No To Shinkei, 56:403-13.

Horton JC. 2005. Vision restoration therapy: confounded by eye movements. Br J Ophthalmol, 89:792-4.

Huber A. 1992. Homonymous hemianopia. Neuro-Ophthalmology, 12:351-66.

Jamara RJ, Van De Velde F, Peli E. 2003. Scanning eye movements in homonymous hemianopia documented by scanning laser ophthalmoscope retinal perimetry. Optom Vis Sci, 80:495-504.

Julkunen L, Tenovuo O, Jääskeläinen S, et al. 2003. Rehabilitation of chronic post-stroke visual field defect with computer-assisted training. Restor Neurol Neurosci, 21:19-28.

Julkunen L, Tenovuo O, Vorobyev V, et al. 2006. Functional brain imaging, clinical and neurophysiological outcome of visual rehabilitation in a chronic stroke patient. Restor Neurol Neurosci, 24:123-32.

Kasten E, Bunzenthal U, Müller-Oehring E, et al. 2007. Vision restoration therapy does not benefit from costimulation: A pilot study. J Clin Exp Neuropsychol, 29:569-84.

Kasten E, Bunzenthal U, Sabel BA. 2006. Visual field recovery after vision restoration therapy (VRT) is independent of eye-movements: An eyetracker study. Behav Brain Res, 175:18-26.

Kasten E, Müller-Oehring E, Sabel BA. 2001. Stability of visual field enlargements following computer-based restitution training - results of a follow-up. J Clin Exp Neuropsychol, 23:297-305.

Kasten E, Poggel DA, Müller-Oehring E, et al. 1999. Restoration of vision II: Residual functions and training-induced visual field enlargement in brain-damaged patients. Restor Neurol Neurosci, 15:273-87.

Kasten E, Poggel DA, Sabel BA. 2000. Computer-based training of stimulus detection improves colour and simple pattern recognition in the defective field of hemianopic subjects. $J$ Cogn Neurosci, 12:1001-12.

Kasten E, Sabel BA. 1995. Visual field enlargement after computer training in brain-damaged patients with homonymous deficits: an open pilot trial. Restor Neurol Neurosci, 8:113-27.

Kasten E, Strasburger H, Sabel BA. 1997. Programs for diagnosis and therapy of visual field deficits in vision rehabilitation. Spat Vis, 10:499-503.

Kasten E, Wüst S, Behrens-Baumann W, et al. 1998. Computer-based training for the treatment of partial blindness. Nature Med, 4:1083-87.

Kedar S, Zhang X, Lynn MJ, et al. 2006. Pediatric Homonymous Hemianopia. JAAPOS, 10:249-52.

Kerkhoff G. 1999. Restorative and compensatory therapy approaches in cerebral blindness - a review. Restor Neurol Neurosci, 15:255-71.

Kerkhoff G. 2000. Neurovisual rehabilitation: recent developments and future directions. J Neurol Neurosurg Psychiatry, 68:691-706.

Kerkhoff G, Münßinger U, Haaf E, et al. 1992. Rehabilitation of homonymous scotomata in patients with postgeniculate damage of the visual system: saccadic compensation training. Restor Neurol Neurosci, 4:245-54.

Kerkhoff G, Münßinger U, Meier EK. 1994. Neurovisual rehabilitation in cerebral blindness. Arch Neurol, 51:474-81.

Kooijman AC, Brouwer WH, Coeckelbergh TRM, et al. 2004. Compensatory viewing training improves practical fitness to drive of subjects with impaired vision. Vis Impair Res, 6:1-27.

Lee AG, Perez AM. 1999. Improving awareness of peripheral visual field using sectorial prism. J Am Optom Assoc, 70:624-8. 
Leff A. 2004. A historical review of the representation of the visual field in primary visual cortex with special reference to the neural mechanisms underlying macular sparing. Brain Lang, 88:268-78.

Leff AP, Crewes H, Plant GT, et al. 2001. The functional anatomy of single-word reading in patients with hemianopic and pure alexia. Brain, 124:510-21.

McDonald SA, Spitsyna G, Shillcock RC, et al. 2006. Patients with hemianopic alexia adopt an inefficient eye movement strategy when reading text. Brain, 129:158-67.

McFadzean RM. 2006. NovaVision: vision restoration therapy. Curr Opin Ophthalmol, 17:498-503.

Meienberg O, Zangemeister WH, Rosenberg M, et al. 1981. Saccadic eye movement strategies in patients with homonymous hemianopia. Ann Neurol, 9:537-44.

Mohler CW, Wurtz RH. 1977. Role of striate cortex and superior colliculus in visual guidance of saccadic eye movements in monkeys. J Neurophysiol, 40:74-94.

Mueller I, Poggel DA, Kenkel S, et al. 2003. Vision restoration therapy after brain damage: Subjective improvements of activities of daily life and their relationship to visual field enlargements. Vis Impair Res, 5:157-78.

Nelles G, Esser J, Exkstein A, et al. 2001. Compensatory visual field training for patients with hemianopia after stroke. Neurosci Lett, 306:189-92.

Pambakian A, Currie J, Kennard C. 2005. Rehabilitation strategies for patients with homonymous visual field defects. J Neuro-Ophthalmol, 25:136-42.

Pambakian ALM, Kennard C. 1997. Can visual function be restored in patients with homonymous hemianopia? Br J Ophthalmol, 81:324-8.

Pambakian ALM, Mannan SK, Hodgson TL, et al. 2004. Saccadic visual search training: a treatment for patients with homonymous hemianopia. J Neurol Neurosurg Psychiatry, 75:1443-8.

Pambakian ALM, Wooding DS, Patel N, et al. 2000. Scanning the visual world: a study of patients with homonymous hemianopia. $J$ Neurol Neurosurg Psychiatry, 69:751-9.

Patel AT, Duncan PW, Lai SM, et al. 2000. The relation between impairments and functional outcomes poststroke. Arch Phys Med Rehabil, $81: 1357-63$

Payne BR, Lomber SG, Macneil MA, et al. 1996. Evidence for greater sight in blindsight following damage of primary visual cortex early in life. Neuropsychologia, 34:741-74.

Pelak VS, Dubin M, Whitney E. 2007. Homonymous hemianopia: A critical analysis of optical devices, compensatory training, and NovaVision. Curr Treat Opt Neurol, 9:41-7.

Peli E. 2000. Field expansion for homonymous hemianopia by optically induced peripheral exotropia. Optom Vis Sci, 77:453-64.

Peli E. 2001. Vision multiplexing: an engineering approach to vision rehabilitation device development. Optom Vis Sci, 78:304-15.

Plant GT. 2005. A work out for hemianopia. Br J Ophthalmol, 89:2.

Pleger B, Foerster A-F, Widdig W, et al. 2003. Functional magnetic resonance imaging mirrors recovery of visual perception after repetitive tachistoscopic stimulation in patients with partial cortical blindness. Neurosci Lett, 335:192-6.

Poggel DA, Kasten E, Müller-Oehring EM, et al. 2006. Improving residual vision by attentional cueing in patients with brain lesions. Brain Res, 1097:142-8.

Reding MJ, Potes E. 1988. Rehabilitation outcome following initial unilateral hemispheric stroke: life table analysis approach. Stroke, 19:1354-58.

Reinhard J, Schreiber A, Schiefer U, et al. 2005. Does visual restitution training change absolute homonymous visual field defects? A fundus controlled study. Br J Ophthalmol, 89:30-5.

Sabel BA, Kenkel S, Kasten E. 2004. Vision restoration therapy (VRT) efficacy as assessed by comparative perimetric analysis and subjective questionnaires. Restor Neurol Neurosurg Neurosci, 22:399-420.
Sànchez-Blanco I, Ochoa-Sangrador C, López-Munaín L, et al. 2002. Predictive model of functional independence in stroke patients admitted to a rehabilitation programme. Clin Rehabil, 13:464-75.

Schreiber A, Vonthein R, Reinhard J, et al. 2006. Effect of visual restitution training on absolute homonymous scotomas. Neurology, 67:143-5.

Schulte T, Strasburger H, Muller-Oehring, et al. 1999. Automobile driving performance of brain-injured patients with visual field defects. Am J Phys Med Rehabil, 78:136-42.

Spitzyna GA, Wise RJS, McDonald SA, et al. 2007. Optokinetic therapy improves text reading in patients with hemianopic alexia: A controlled trial. Neurology, 68:1922-30.

Stelmack J. 2001. Quality of life of low-vision patients and outcomes of low vision rehabilitation. Optom Vis Sci, 78:335-42.

Szlyk JP, Seiple W, Stelmack J, et al. 2005. Use of prisms for navigation and driving in hemianopic patients. Ophthal Physiol Opt, 25:128-35.

Trauzettel-Klosinski S. 1997. Eccentric fixation with hemianopic field defects: A valuable strategy to improve reading ability and an indication of cortical plasticity. Neuro-Ophthalmology, 18:117-31.

Trauzettel-Klosinski S, Brendler K. 1998. Eye movements in reading with hemianopic field defects: the significance of clinical parameters. Graefes Arch Clin Exp Ophthalmol, 236:91-102.

Verlohr D, Dannheim F. 2007. The visual performance test: indications for compensational visual rehabilitation training and first results. Strabismus, 15:63-8.

Weiskrantz L, Warrington EK, Sanders MD, et al. 1974. Visual capacity in the hemianopic field following a restricted occipital ablation. Brain, 97:709-28.

Werth R, Moehrenschlager M. 1999. The development of visual functions in cerebrally blind children during a systematic visual field training. Restor Neurol Neurosci, 15:229-41.

Werth R, Seelos K. 2005. Restitution of visual functions in cerebrally blind children. Neuropsychologia, 43:2011-23.

Zhang X, Kedar S, Lynn MJ, et al. 2006. Natural history of homonymous hemianopia. Neurology, 66:901-5.

Zihl J. 1980. "Blindsight": Improvement of visually guided eye movements by systematic practice in patients with cerebral blindness. Neuropsychologia, 18:71-7.

Zihl J. 1995a. Visual scanning behavior in patients with homonymous hemianopia. Neuropsychologia, 33:287-303.

Zihl J. 1995b. Eye movement patterns in hemianopic dyslexia. Brain, 118:891-912.

Zihl J. 1999a. Cerebral Disturbances of Elementary Visual Functions. In: Brown JW (ed). Neuropsychology of Visual Perception. New York: Lawrence Erlbaum Associates, Inc. pp. 35-58.

Zihl J. 1999b. Oculomotor scanning performance in subjects with homonymous visual field disorders. Vis Impair Res, 1:23-31.

Zihl J. 2000. Rehabilitation of Visual Disorders After Brain Injury. East Sussex: Psychology Press Ltd.

Zihl J, Kennard C. 1996. Disorders of Higher Visual Function. In: Brandt T, Caplan LR, Dichgans J, et al eds. Neurological Disorders: Course and Treatment. California: Academic Press. pp. 201-12.

Zihl J, Roth W, Kerkhoff G, et al. 1988. The influence of homonymous visual field disorders on colour sorting performance in the FM 100-hue test. Neuropsychologia, 26:869-76.

Zihl J, Von Cramon D. 1979. Restitution of visual function in patients with cerebral blindness. J Neurol Neurosurg Psychiatry, 42:312-22.

Zihl J, Von Cramon D. 1985. Visual field recovery from scotoma in patients with postgeniculate damage: a review of 55 cases. Brain, 108:335-65.

Zihl J, Wohlfarth-Englert A. 1986. The influence of visual field disorders on visual identification tasks. Eur Arch Psychiatry Neurol Sci, 236:61-4 